\title{
LOCALLY TRIVIAL HOMOLOGY THEORIES, AND THE POINCARE DUALITY THEOREM
}

\author{
BY H. B. GRIFFITHS
}

Communicated by Deane Montgomery, July 21, 1958

The following is a brief account of a forthcoming memoir. A complex with covering is a triple $(K, \mathcal{K}, \mathrm{St})$, of ten written $(K, \mathfrak{K})$, where (i) $K$ is a chain complex $\left\{C_{q} K, \partial_{q}\right\}$ and $\Re=\left\{K^{\lambda}\right\}$ is a collection of subcomplexes of $K$ such that $K=\Sigma K^{\lambda}$, i.e. each $x \in C_{q} K$ is a finite sum of members of the groups $C_{q} K^{\lambda}, K^{\lambda} \in \Re$; (ii) $K$ is augmented, i.e. $\partial_{0}$ is a homomorphism of $C_{0} K$ in the integers, such that $\partial_{0} \mid C_{0} K^{\lambda}$ is onto, for each $K^{\lambda}$; (iii) each $K^{\lambda}$ lies in some sub-complex St $K^{\lambda}$ of $K$, (e.g. St $K^{\lambda}$ might be $\Sigma K^{\mu}$ over all $\mu$ with $K^{\lambda} \cap K^{\mu}$ nontrivial). (K, $\mathscr{K}$ ) is free whenever there exist sets $G_{q}$ such that $G_{q} \cap C_{q} K^{\lambda}$ freely generates each $K^{\lambda},(0 \leqq q \leqq \infty)$.

Let $\left(K, \mathfrak{K}, \mathrm{St}_{K}\right),\left(J, \mathcal{g}, \mathrm{St}_{J}\right)$ be complexes with covering. A map $\pi: \mathfrak{K} \rightarrow \mathcal{J}$ is coherent whenever $K^{\lambda} \cap K^{\mu}$ nontrivial implies $\pi K^{\lambda}$ $\subseteq \mathrm{St}_{J}\left(\pi K^{\mu}\right)$. A relation $(K, \mathscr{K}) \rightarrow^{u}(J, g)$ is a chain homomorphism $u_{1}: K \rightarrow J$ which preserves augmentations, together with a map $u_{2}: \mathcal{K} \rightarrow \mathcal{J}$, such that $\operatorname{Im}\left(u_{1} \mid K^{\lambda}\right) \subseteq u_{2} K^{\lambda}$ for all $K^{\lambda} \in \mathcal{K}$. We replace the arrow in the above relation by $\rightarrow_{*}$ or $\rightarrow_{q}$ according as $\operatorname{Im}\left(u_{1} \mathrm{St}_{K} K^{\lambda}\right)$ $\subseteq u_{2} K^{\lambda}$, or $\operatorname{Im}\left(u_{1} \mid q\right.$-cycles of $\left.K^{\lambda}\right) \subseteq$ boundaries of $u_{2} K^{\lambda}$, for each $K^{\lambda} \in \mathcal{K}$.

Now suppose there exists a diagram of relations

$$
\begin{aligned}
& \left(A^{0}, Q^{0}\right) \stackrel{u}{\rightarrow}(A, Q) \stackrel{v}{\rightarrow}\left(B^{1}, B^{1}\right) \underset{0}{\longrightarrow}\left(A^{1}, Q^{1}\right) \rightarrow \rightarrow_{*}^{\rightarrow} \cdots
\end{aligned}
$$

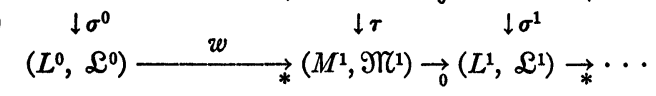

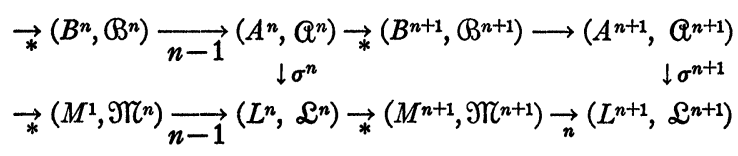

(the top right arrow carrying no " $n$ "). Suppose that $\left(L^{0}, \mathscr{L}^{0}\right)$ is free, and that there exists a coherent map $\gamma: \mathscr{L}^{0} \rightarrow \mathbb{Q}$ such that (i) the composite maps $\mathfrak{L}^{0} \rightarrow \mathfrak{Q}^{q}, \mathfrak{L}^{0} \rightarrow \mathfrak{L}^{q}$ are coherent; (ii) $\gamma \sigma_{2}^{0}=u_{2}^{0}$; (iii) $\tau v_{2} \gamma=w_{2}$; (iv) each square is commutative (e.g. $\tau_{i} v_{i} u_{i}=w_{i} \sigma_{i}^{0}, i=1,2$ ). Then for each $q=0,1, \cdots, n+1$, and abelian group $G$, there exist homology and cohomology diagrams 
$(\dagger \dagger)$

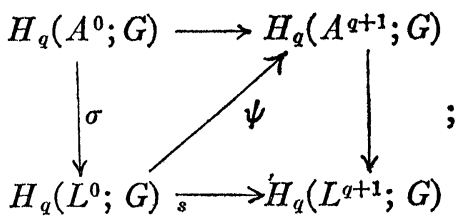

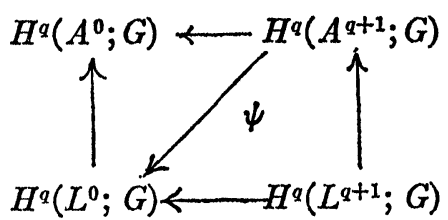

which are everywhere commutative, except above the diagonal if $q=n+1$. The vertical and horizontal maps are induced by corresponding ones in the diagram $(\dagger)$, and the $\psi$ 's by constructing a map (by induction on $q$ ) at chain level, and then applying the functors $\otimes G, \operatorname{Hom}(\cdot, G)$.

EXAmple (1). Let $f: Y \rightarrow X$ be a map of metric spaces, and let us prove a Vietoris-type mapping theorem. Let $S_{\epsilon} X$ be that subcomplex of the singular complex of $X$ which is generated by all cells of diameter $<\epsilon$. If $\{U\}$ is an open covering of $X$, then $S_{\epsilon}(X)$ is covered by $\left\{S_{\epsilon} U\right\}$, and we define St $\left(S_{\epsilon}(U)\right)$ to be $S_{\epsilon}$ (St $\left.U\right)$, St $U=$ star of $U$ in $\{U\}$. Taking the augmentation which is 1 on all zero-cells, $\left(S_{\epsilon} X\right.$, $\left.\left\{S_{\epsilon} U\right\}, \mathrm{St}\right)$ is a free complex with covering, and similarly so is $\left(S_{\epsilon} Y,\left\{S_{\epsilon} f^{-1} U\right\}, \mathrm{St}\right)$; further, if $\{V\}$ star-refines $\{U\}$ in $X$, then there is an obvious relation $\left(S_{\epsilon} X,\left\{S_{\epsilon} V\right\}\right) \rightarrow_{*}\left(S_{\epsilon} X,\left\{S_{\epsilon} U\right\}\right)$. Since $X$ is paracompact, every $\{U\}$ has a star refinement, and so a suitable sequence of coverings $\{U\}$ can be found to yield a diagram of the form $(\dagger)$, the $\sigma$ 's being induced by $f$,-provided that $X$ is singularly locally connected in dimensions up to $n+1$, and the fibres of $f$ have a suitable acyclicity property of their neighborhoods in $Y$; this proviso enables one to construct the relations of the form $\rightarrow_{q}$. Turning then to the diagrams $(\dagger \dagger)$, one has $L^{0}=L^{q+1}=S_{\epsilon} X$ for some $\epsilon$, so that $s$ there, for example, is the identity isomorphism; $\sigma$ is $f_{* q}$, and $H S_{e}$ $=H X$. Hence $f_{* q}: H_{q}(Y ; G) \rightarrow H_{q}(X ; G)$ is an isomorphism, $0 \leqq q \leqq n$, and $f_{*, n+1}$ is onto; similarly for cohomology. The result holds for maps of paracompact, or of locally compact, spaces.

Example (2). Poincaré duality in Cech theory with integer coefficients and a locally compact space. The known sheaf-theoretic forms (cf. Borel, Michigan Math. J. vol. 4 (1957)) of this result involve a group $H_{q}($ Hom(Alexander cochains)), which is not usually known unless coefficients form a field. The following result shows what the group must be, in the problem at hand. Let $R$ be locally compact, with compactification $X=R+\infty$ of covering dimension $n$. Using the Cech functor $H$, assume $H_{n}(X)=H^{n}(X)=H_{0}(X)=$ integers. Define

$$
\begin{aligned}
& H_{c}^{q}(R)=H^{q}(X, \infty)=\operatorname{Dlim}\left\{H^{q}(X, X-G)\right\} ; \\
& H_{c q}(R)=\operatorname{Dlim}\left\{H_{q}(\mathrm{Cl} G)\right\},
\end{aligned}
$$


the direct-limits of groups and injections being indexed by the set $T$ of all open $G \subseteq R$ with compact closure $\mathrm{Cl} G$. Fix $q_{0}, 0 \leqq q_{0} \leqq n$. Assume that locally, each $x \in R$ has a basis $B$ of open neighborhoods, such that, given $P \in B$, and a sufficiently small $Q \in B$, then the diagram

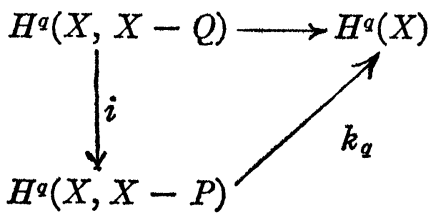

of injections satisfies: $i$ trivial $\left(q_{0} \leqq q \leqq n\right), k_{n} \mid \operatorname{Im} i$ an isomorphism on $H^{n}(X)$. Let $\Gamma$ generate $H_{n}(X)$.

THEOREM. The cap product with $\Gamma$ induces a map $\theta_{q}: H_{c}^{q}(R) \rightarrow H_{c, n-q}(R)$ which is an isomorphism if $q_{0} \leqq q \leqq n$, and onto if $q=q_{0}-1$.

(Compare Čech, Proc. Nat. Acad. Sci. U.S.A. vol. 22 (1936) p. 110). The map $\theta_{q}$ is natural relative to maps of $X$, except when $q=n$, when the maps need to be injections. This arises because $\theta_{n}$ has kernel zero (since it has "over the rationals," and there is no homology $n$-torsion), and we change coefficients of $H_{c 0}(R)$ to be multiples of $\xi$, where $\operatorname{Im} \theta_{n}=\xi \cdot H_{c 0}(R)$; we do not know if $\xi$ must be 1 . This change does not affect the other homology groups, and makes $\theta_{n}$ onto. The proof of the theorem follows from the diagrams $(\dagger)$ and $(\dagger \dagger)$ above, as follows. We eventually construct diagrams $D_{1}, D_{2}$ :

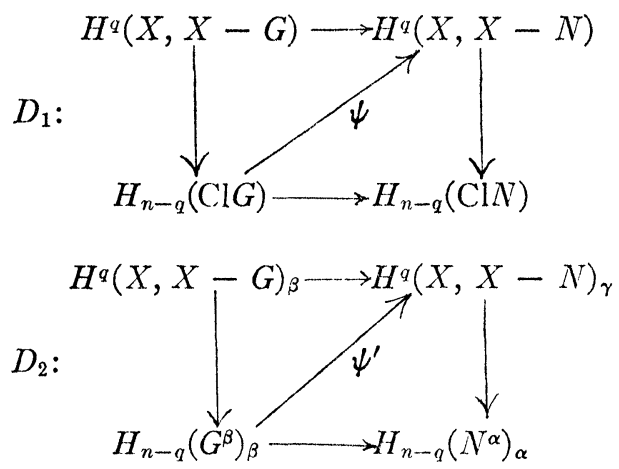

explaining the notation as required. If $D_{1}$ exists with $\mathrm{Cl} G \subseteq N \in T$, the horizontal arrows induced by inclusion and the verticals by the cap product, then the required statement about $\theta_{q}$ follows from the commutativity properties, by taking a direct limit over $T$ of diagrams like $D_{1}$. To construct $D_{1}$, we take limits of diagrams like $D_{2}$, over the set Cov $X$ of finite open coverings of $X$, directed by refinement; and to 
see this we explain $D_{2}$. It exists for any $\alpha \in \operatorname{Cov} X$, and sufficiently fine $\beta, \gamma$, with $\alpha \leqq \beta \leqq \gamma$ in $\operatorname{Cov} X$. If $A \subseteq X$, then $A^{\alpha}$ is the star of $A$ in $\alpha$, and $A_{\alpha}$ the subcomplex of Nerve ( $\alpha$ ) generated by all cells whose support meets $A ;(X, X-G)_{\beta}=\left(X_{\beta},(X-G)_{\beta}\right)$. Then $\mathrm{Cl} G=\cap G^{\beta}$, $\beta \in \operatorname{Cov} X$, so that the groups $H_{n-q}\left(G^{\beta}\right)_{\beta}$ and obvious maps, have by continuity an inverse limit $H_{n-q}(\mathrm{Cl} G)$; also $H^{q}(X, X-G)$ $=\operatorname{Dlim}\left(H^{q}(X, X-G)_{\beta}\right)$ (with obvious maps). To say that $\psi=\lim \psi^{\prime}$, $\left(\psi^{\prime}=\psi^{\prime}(\alpha, \beta, \gamma)\right)$, it is necessary to check certain commutativity relations, and this can be done. Thus, it remains to construct $D_{2}$. For this, we use the cochains and chains of suitable nerves for the complexes in the top and bottom lines of $(\dagger)$, respectively; and make coverings for them with coverings of $N$, rather as in Example 1. The relations $\rightarrow_{q}$ follow for the cochains by $(\dagger \dagger \dagger)$ and for the chains from the fact that if $A \in \alpha \in \operatorname{Cov} X$, then $A_{\alpha}$ is a cone with acyclic homology. The relations $\rightarrow_{*}$ follow as in Example 1, as does the augmentation for the chains. That for the cochains is the composite map

$$
C^{n}\left(X_{\alpha}\right) \rightarrow H^{n}\left(X_{\alpha}\right) \rightarrow H^{n}(X) \stackrel{\theta_{n}}{\rightarrow} H_{0}(X)=\text { integers. }
$$

The conditions (i)-(iv) imposed on ( $\dagger$ ) are then verified, and so $D_{2}$ exists, as required.

Other interpretations of the diagram ( $\dagger$ ) give the De Rham theorem and a uniqueness theorem for singular homology, as well as known theorems on pairs of coverings, and technical lemmas. Consequences of the duality theorem are Alexander duality and Wilder's theorem (Pacific J. Math. vol. 7 (1957)), with integer coefficients. 